\title{
A CONSTITUTIVE LAW WITH IMPROVED CONTINUOUS-DISCONTINUOUS DESCRIPTION OF CRACKS IN CONCRETE
}

\author{
JERZY BOBIŃSKI ${ }^{*}$ AND JACEK TEJCHMAN ${ }^{\dagger}$ \\ Gdansk University of Technology (GUT) \\ 80-233 Gdansk, Narutowicza 11/12, Poland \\ e-mail: bobin@pg.gda.pl \\ ${ }^{\dagger}$ Gdansk University of Technology (GUT) \\ 80-233 Gdansk, Narutowicza 11/12, Poland \\ e-mail: tejchmk@pg.gda.pl
}

Key words: Concrete, Crack, Non-local plasticity, Extended Finite Element Method (XFEM)

\begin{abstract}
Paper presents an improved version of the constitutive model which combines a continuous and discontinuous crack's description to simulate the concrete under tensile dominated loads. In a continuum regime, a plasticity model with a Rankine failure criterion and an associated flow rule was used. To describe the width of strain localization and to obtain mesh-independent results, the continuum constitutive law was equipped with a characteristic length of micro-structure by applying a non-local theory in an integral format. For describing displacement jumps along/across cracks, the eXtended Finite Element Method (XFEM) was used. A transition algorithm between a non-local continuum model and XFEM was formulated. A transfer function was introduced to enable a gradual switch from a continuous (smeared) to discontinuous (discrete) softening process. Several benchmarks were numerically simulated with a dominated mode-I (e.g. uniaxial tension and bending) and under mixed-mode conditions.
\end{abstract}

\section{INTRODUCTION}

Fracture in concrete is responsible for the both strength and stiffness reduction and it precedes the structural failure. At the beginning of loading, a region with several micro-cracks is formed. Later these microcracks create a macro-crack. An adequate description of fracture in numerical $\mathrm{FE}$ calculations is extremely important to obtain physically realistic results. Within continuum mechanics, there exist two main approaches to describe fracture. The first one describes it in a smeared sense as localized zones of microcracks with a certain finite width. Since this formulation includes softening, it has to be equipped with a characteristic length of microstructure to preserve the well-posedness of the boundary value problem. It can be done by means of e.g. a micro-polar, non-local or gradient theories. As an alternative, displacement jumps (discontinuities) along cracks may be introduced while keeping the remaining region as a continuous one. The oldest solutions used interface elements defined along element edges. The modern ones allow for considering cracks in the interior of finite elements using embedded discontinuities or XFEM (eXtended Finite Element Method) [1] based on a concept of the partition of unity. A smeared approach is more appropriate when describing a micro-crack formation process while a discontinuous one allows for a more realistic simulation of discrete macro-crack propagation. Usually, only one approach is used to simulate 
a fracture process in concrete during the entire deformation process. A combination of continuous and discontinuous approaches make it possible (as in experiments) to realistically capture all fracture stages. Such a coupling can be done in several ways, e.g. the latest approaches combine XFEM with implicit gradient [2] or integral-nonlocal [3] isotropic damage models. Wells et al. [4] combined XFEM with the Perzyna viscoplastic model.

The continuous-discontinuous constitutive law presented here is an improved version of the model earlier formulated $[5,6]$. The original one links a continuous and discontinuous description of fracture defined within continuum mechanics. In a continuum regime, an elasto-plastic constitutive law with a Rankine criterion and an associated flow rule is used. In order to restore the well-posedness of the boundary value problem it is equipped with a characteristic length using an integral non-local theory. Discontinuous displacement jumps are described with XFEM. Discrete cracks are assumed to follow smeared crack patterns instead of using criteria based on a stress distribution. An special algorithm is responsible for transferring a softening from bulk points into a newly created crack segments. The proposed extension introduces a transfer function which allows for a gradual switch from a continuous (smeared) to discontinuous (discrete) softening process. By extending a crack with a new segment, nodes and finite elements located in a band perpendicular to this segment are doubled. The width of a doubled zone covers the width of a localized zone. Both doubled element sets share the same nodes along zone boundaries. As a consequence no special algorithm is required to force transfer of the softening mechanism. Such a modification overcomes some convergence problems observed in simulations with original models. The effectiveness of the proposed formulation is verified on several tests including uniaxial stress states (tension and bending tests) and multiaxial stress conditions (the so-called Nooru-Mohamed problem).

\section{CONTINUOUS APPROACH}

\subsection{Elasto-plasticity}

In continuum, an elasto-plastic constitutive law with the standard Rankine criterion was used. The yield function for 2D case was defined as:

$$
f=\max \left\{\sigma_{1}, \sigma_{2}\right\}-\sigma_{t}\left(\kappa_{p}\right),
$$

where $\sigma_{1}$ and $\sigma_{2}$ - the principal stresses, $\sigma_{t}-$ the tensile yield stress and $\kappa_{p}-$ the hardening/softening parameter (equal to the maximum principal plastic strain $\varepsilon_{1}^{p}$ ). An associated flow rule was assumed. To define softening under tension, two alternative curves: a linear or an exponential were used. The first one was defined as:

$$
\sigma_{t}\left(\kappa_{p}\right)=\max \left\{0, f_{t}\left(1-\frac{\kappa_{p}}{\kappa_{u}}\right)\right\},
$$

where $f_{t}$ - the tensile strength and $\kappa_{u}-$ the ultimate value of the softening parameter $\kappa_{p}$. The exponential curve was chosen as:

$$
\sigma_{t}\left(\kappa_{p}\right)=f_{t} \exp \left(-\frac{\kappa_{p}}{\kappa_{u}}\right) .
$$

Here the parameter controls the slope of a softening curve (it can be interpreted as an intersection point of a tangent line from the peak point with the horizontal axis). A formulation with this simple exponential softening curve produces very similar results to ones with more sophisticated curves, e.g. the experimentally motivated non-linear curve by Hordijk [7].

\subsection{Non-locality}

Strain softening causes the ill-posedness of the boundary value problem and FE-results become mesh dependent. In order to achieve mesh-independent results, a non-local theory in an integral format was used as a regularization technique. It is based on a spatial averaging of tensor or scalar state variables in a certain neighborhood of a given material point. The rates of the softening parameter $\mathrm{d} \kappa_{p}$ were treated non-locally 
according to Brinkgreve [8]:

$$
\mathrm{d} \overline{\boldsymbol{\kappa}}_{p}(\boldsymbol{x})=(1-m) \mathrm{d} \boldsymbol{\kappa}_{p}(\boldsymbol{x})+m \mathrm{~d} \hat{\boldsymbol{\kappa}}_{p}(\boldsymbol{x})
$$

with:

$$
\mathrm{d} \hat{\kappa}(x)=\frac{\int_{V} \alpha_{0}(\|x-\xi\|) \mathrm{d} \kappa_{p}(\xi) \mathrm{d} \xi}{\int_{V} \alpha_{0}(\|x-\xi\|) \mathrm{d} \xi}
$$

where $\boldsymbol{x}$ - the coordinates of considered (actual) point, $\xi$ - the coordinates of the surrounding points, $m$ - the non-locality parameter (it should be greater than 1). As the weighting function $\alpha_{0}$, the Gauss distribution was assumed:

$$
\alpha_{0}(r)=\frac{1}{l \sqrt{\pi}} e^{-\left(\frac{r}{l}\right)^{2}}
$$

where $r=\|\boldsymbol{x}-\boldsymbol{\xi}\|$ - the distance between points $\boldsymbol{x}$ and $\boldsymbol{\xi}$ and $l-$ the characteristic length of the microstructure. The above averaging was restricted only to a small area around each material point (the influence of points at the distance of $r=3 l$ is only of $0.01 \%$ ). Therefore despite a theoretical unbounded support of the weighting function $\alpha_{0}$, the bounded (limited) support could be assumed for practical considerations.

In FE-simulations, an approximated method was used to evaluate non-local quantities. In the given integration point, the influence of its neighbors was determined using the values from the previous iteration. It enabled us to simplify FE calculations and to preserve the locality of plasticity algorithms

\section{DISCONTINUOUS APPROACH}

\subsection{General formulation}

In order to describe displacement jumps in continuum along cracks, the Extended Finite Element Method (XFEM) was chosen. It is based on a local partition of the unity (PUM) concept by Melenk and Babuška [9]. It enables adding 'ad hoc' extra terms to a standard FE displacement field interpolation. These extra functions are responsible for capturing displacement jumps. In XFEM cracks do not have to be placed along finite element edges; they may pass through elements without remeshing. Due to a local nature of the enrichment, extra displacement degrees of freedom are solely introduced into finite elements cut by a discontinuity. They are gradually added to subsequent finite elements when a crack propagates. The formulation used in the paper follows (with some improvements and modifications) the original concept by Wells and Sluys [10]. In order to describe jumps in the displacement field, the so-called shifted-basis enrichment was used [11]. The shifted-basis enrichment simplifies the implementation of XFEM (two types of the finite elements exist only). Moreover the total nodal displacements are equal to the standard displacements.

In the bulk continuum, a linear elastic relationship was assumed. An extra constitutive law between displacement jumps $[[\boldsymbol{u}]]$ and tractions $t$ was defined in the crack. The degradation process was controlled by the variable $\kappa_{x}$ defined as a maximum value of normal displacement jumps $\left[\left[u_{n}\right]\right]$ obtained during loading. During active loading the softening of the normal component of the traction vector $t_{n}$ was described by the yield curve $\sigma_{n}$ using either a linear relationship:

$$
\sigma_{n}=D_{f} f_{t}\left(1-\frac{f_{t} \kappa_{x}}{2 G_{f}}\right)
$$

or an exponential one:

$$
\sigma_{n}=D_{f} f_{t} \exp \left(-\frac{f_{t} \kappa_{x}}{G_{f}}\right),
$$

where $G_{f}$ - the fracture energy and $D_{f}$ - the correction term defined as:

$$
D_{f}=1-\exp \left(-\kappa_{x} \frac{d_{f} f_{t}}{G_{f}}\right),
$$

where $d_{f}$ - the drop factor [12]. This factor improves the convergence in cases with tension-compression changes. With increasing $d_{f}$, the term $D_{f}$ approaches 1. During unloading, the secant stiffness was used with a return to the origin (damage format). In a compressive regime, the penalty stiffness in 
the normal direction was used (it depends on the drop factor $d_{f}$ ). In a tangential direction the following formula was assumed:

$$
t_{s}=T_{s} \frac{\sigma_{n}}{f_{t}}\left[\left[u_{s}\right]\right]
$$

with the initial shear stiffness $T_{s}$. With decreasing normal yield traction $\sigma_{n}$, the shear traction $t_{s}$ also decreases (due to the decrease of the resultant shear stiffness).

\subsection{Crack growth algorithm}

In the 'pure' XFEM a new crack could be activated or an existing crack could propagate, if the standard Rankine criterion $\left(\sigma_{\max }>f_{t}\right)$ was fulfilled at least in one point of the finite element at the front of a crack tip. In the extended version of the activation criterion, this inequality was examined not only in the integration points in this element, but also in all integration points in a circle section with the radius $l_{c r k}$ and angle $\varphi_{c r k}$ at the front of the crack tip. This section was symmetrically located along the extension of the last crack segment (Fig.1).

a)

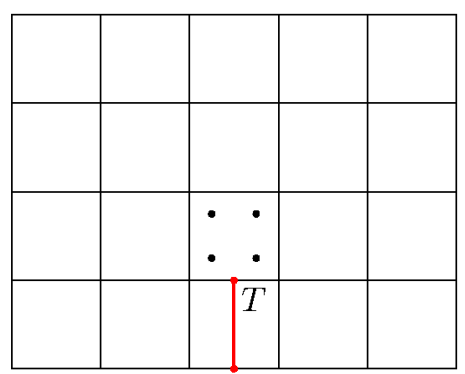

b)

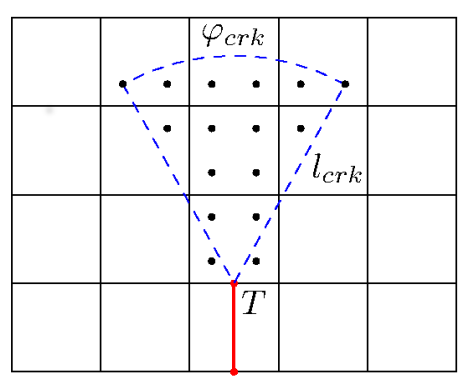

Figure 1: Examination of crack activation criterion: a) standard method and b) extended method.

The direction of the crack propagation usually is determined by analyzing the stress field at the crack tip front. Here this direction was assumed to be perpendicular to the direction of the maximum principal stress. In order to smoothen the stress field around the crack tip, the average stresses $\sigma^{*}$ was used for determining the crack direction according to Wells and Sluys [10]:

$$
\sigma^{*}=\int_{V} \sigma w \mathrm{~d} V
$$

where $V$ - the semi-circle domain at the front of the crack tip and $w$ - the weight function defined as:

$$
w(r)=\frac{1}{(2 \pi)^{3 / 2} l_{a v}^{3}} \exp \left(-\frac{r^{2}}{2 l_{a v}^{2}}\right),
$$

where $l_{a v}$ - the averaging length related to the size of finite elements. Note that this operation is not intended to introduce a length of microstructure into the model. If the direction of the crack propagation is known in advance, a fixed value may be applied. A new crack tip could be placed at the element edge exclusively.

\section{COUPLED APPROACH}

\subsection{Transition process}

Initially, in all finite elements (integration points), a continuum constitutive law with non-local softening was active. A new crack segment was created if the following condition was fulfilled:

$$
\bar{\kappa}_{p}>\kappa_{c d},
$$

where $\kappa_{c d}$ - the softening parameter at the transition moment. This inequality was examined in all integration points in the finite element at the front of the crack tip. Alternatively, the extended version could be used (Fig. 1). If this criterion was fulfilled at least in one integration point, a new crack segment was added. The direction of the new segment $\boldsymbol{p}$ was not derived from the stress field (as in 'pure' XFEM) but it was calculated using the following formula (after [4]):

$$
\boldsymbol{p}(\boldsymbol{x})=\int_{V} \kappa_{p}(\boldsymbol{\xi}) w(\|\overline{\boldsymbol{p}}\|) \frac{\overline{\boldsymbol{p}}}{\|\overline{\boldsymbol{p}}\|} \mathrm{d} V,
$$

where $\overline{\boldsymbol{p}}$ is the direction from the crack tip $\boldsymbol{x}$ to 
any point $\xi$ in $V$ and $w$ denotes the weight function defined by Eq. 12.

Two conditions had to be fulfilled in the formulation of the coupled continuousdiscontinuous constitutive law. The first one is stress equilibrium. Initial tractions $t_{n}^{\text {init }}$ and $t_{s}^{\text {init }}$ were calculated by projecting continuum stresses onto a crack segment line at the crack creation moment. On the basis on the known tractions $t_{n}^{i n i t}$ and $t_{s}^{\text {init }}$, values of initial displacement jumps $\llbracket u_{n}^{\text {init }} \rrbracket$ and $\llbracket u_{s}^{\text {init }} \rrbracket$ were determined using Eqs (7-10), see also Fig. 2. The initial displacement jump $\llbracket u_{n}^{\text {init }} \rrbracket$ was also added to the value of the parameter $\kappa_{x}$. As a consequence, XFEM softening in a normal direction started not from the value $f_{t}$ (peak) but from the smaller value. A newly inserted crack segment had already accumulated softening.

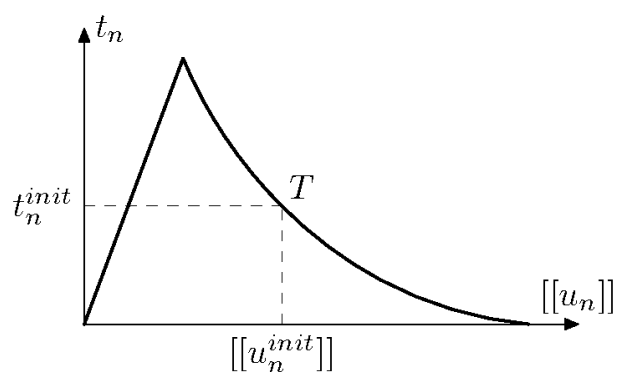

Figure 2: Initial traction and displacement.

The second condition required the equivalence of the dissipated energy. The energy not yet dissipated in the continuum softening should be equal to the energy to be dissipated in the discrete softening process (starting from 'non-peak' configuration). When using elasto-plasticity with non-local softening and XFEM, dissipated energies for the same value of the yield stress are identical and no special modifications of the softening curve in XFEM is required. To ensure the equivalence of the total dissipated energy in the both approaches, it is sufficient to relate fracture energy $G_{f}$ from XFEM with parameters $\kappa_{u}, \quad m$ and $l$ in non-local plasticity [6].

\subsection{Gradual transfer}

A change of the width of the active localization zone from several finite elements in a non-local model to one finite element in XFEM may cause some convergence problems $[5,6]$. To overcome them an improvement was proposed here. The proposed extension introduced a transfer function $\rho$ which allowed for a gradual switch from a continuous (smeared) to discontinuous (discrete) softening process. It was equal to zero at the tip (no discrete softening) and increased to the maximum value of 1 (no continuum softening). The transfer function $\rho$ depended on the value of the XFEM variable $\kappa_{x}$, Fig. 3. Usually the initial value $\kappa_{0}$ corresponded to $\kappa_{x}$ at the transition moment. The $\kappa_{1}$ value controlled the "transition speed".

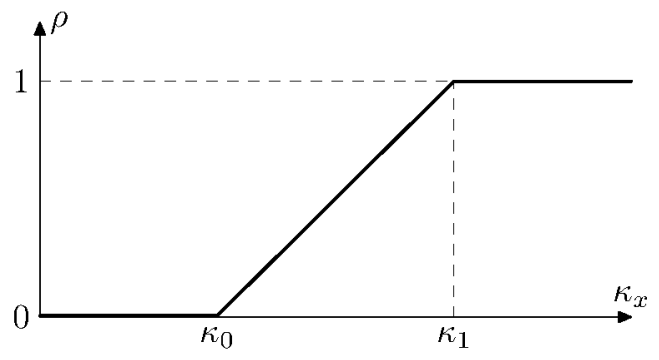

Figure 3: Transfer function $\rho$.

By extending a crack with a new segment, nodes and finite elements located in a band perpendicular to this segment were doubled. The width of a doubled zone covered the width of a localized zone (it was equal to $6 l$ in all simulations). In the bottom layer of finite elements, a continuum law with softening was applied. In the top layer of finite elements, linear elasticity in bulk and a discrete crack with cohesive softening law were used. The resultant stresses $\sigma$ were calculated as:

$$
\sigma=(1-\rho) \sigma_{C^{+}} \rho \sigma_{D}
$$

where $\sigma_{C}-$ the stresses in the bottom (continuous) layer and $\sigma_{D}-$ the stresses in the top (discontinuous) layer. The values of the transfer function $\rho$ were perpendicularly projected from the crack. The doubled element sets shared the same nodes along zone boundaries (Fig. 4a). As a consequence no 
special algorithm was required to force transfer of the softening between both approaches.

An extension of the proposed method with not perpendicular (to the last crack segment) band front was also proposed. The deviation from the perpendicular direction was controlled by the angle $\alpha_{f}$. The isolines of the transfer function $\rho$ were accordingly inclined.

a)

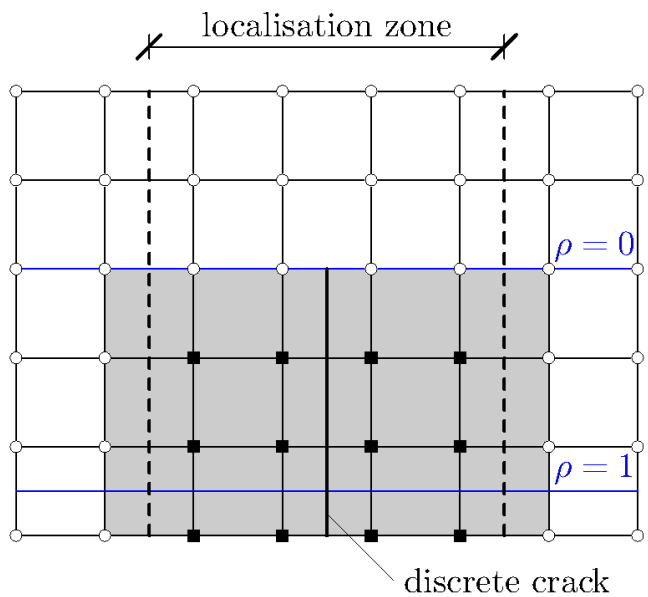

b)

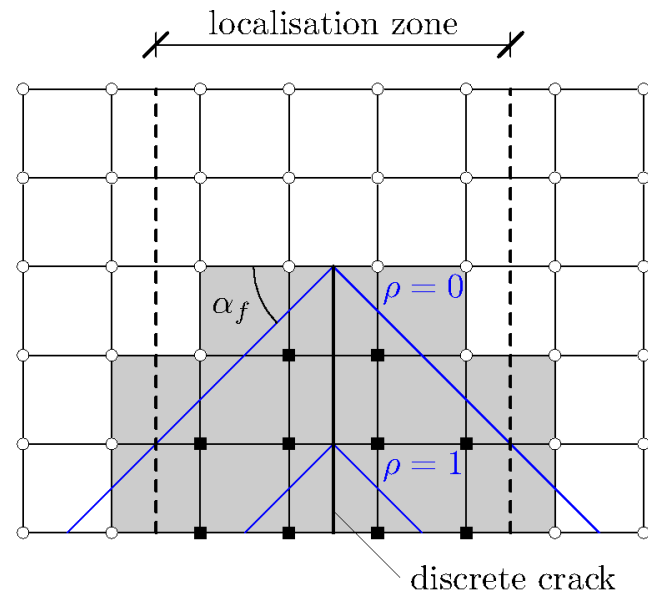

Figure 4: Doubled FE mesh (grey region) with standard (hollow circles) and doubled (black squares) nodes and isolines of function $\rho$ for: a) $\alpha_{f}=90^{\circ}$ and b) $\alpha_{f}<90^{\circ}$.

\section{UNIAXIAL TENSION}

The width of the plane specimen was $100 \mathrm{~mm}$ and height $150 \mathrm{~mm}$ (Fig. 5). All nodes along the bottom were fixed in a vertical direction. The tensile deformation was imposed by enforcing the vertical displacement increment of all nodes along the upper edge by the same amount of $\Delta u=0.1 \mathrm{~mm}$. In order to preserve the specimen stability, the bottom mid-node was fixed in a horizontal direction.
The modulus of elasticity was equal to $E=30$ $\mathrm{GPa}$, Poisson's ratio was $v=0.2$ and tensile strength $f_{t}=3 \mathrm{MPa}$. Linear softening was assumed in the both regimes. The ultimate softening parameter in plasticity was equal to $\kappa_{u}=1.74 \cdot 10^{-3}$. The characteristic length of microstructure was $l=10 \mathrm{~mm}$ and non-locality parameter $m=2$. In order to induce strain localization, a zone of weak elements (with the tensile strength reduced to $2.99 \mathrm{MPa}$ ) was assumed at the specimen mid-height. Its height was equal to $10 \mathrm{~mm}$. In XFEM, the fracture energy was $G f=100 \mathrm{~N} / \mathrm{m}$, drop factor $d_{f}=10^{4}$ and tangential stiffness $T_{S}=10^{12} \mathrm{~N} / \mathrm{m}^{3}$. The simulations were carried out with the transition point in a softening regime equal to $\kappa_{c d}=0.88 \cdot 10-3$ (corresponding to $50 \%$ of the tensile strength $f_{t}$ ). The parameters $\kappa_{0}$ and $\kappa_{1}$ were equal to $3.33 \cdot 10^{-5}$ and $5.0 \cdot 10^{-5}$, respectively (they corresponded to $0.50 f_{t}$ and $0.25 f_{t}$ ). A family of regular FE-meshes was defined. Each mesh consisted of $n_{c}$-columns and $1.5 n_{c}$-rows of finite cells. Each cell was composed of one 4-node quad element. The plane stress conditions were assumed.

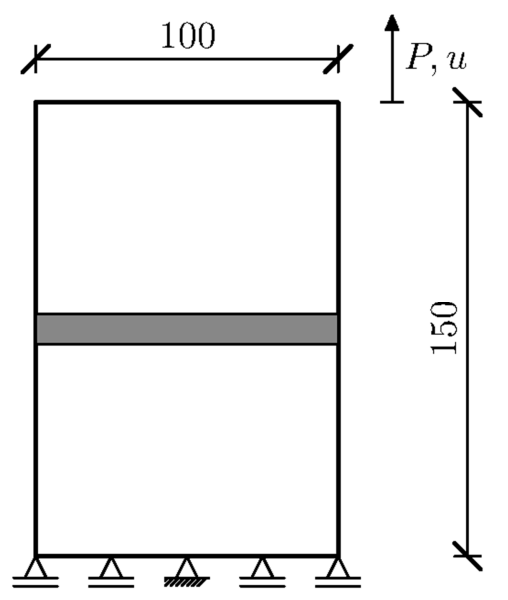

Figure 5: Uniaxial tension: geometry with weak zone location (shaded area) and boundary conditions ( $P$ vertical force along upper edge and $\mathrm{u}$ - vertical displacement of upper edge).

Figure 6 presents the force-displacement diagrams for different meshes. The identical curves were obtained. No jumps could be observed at the moment of the crack creation or when the elasto-plastic model was turned off. Although a general crack growth 
algorithm was applied (with a segment by segment crack elongation), the crack was created in one increment. By default the simulations were performed with the front angle $\alpha_{f}=0^{\circ}$. The calculations were repeated with the different angles $\alpha_{f}$. No influence of the front angle was observed in the uniaxial tension; the same force-displacement curves were obtained (Fig. 7).

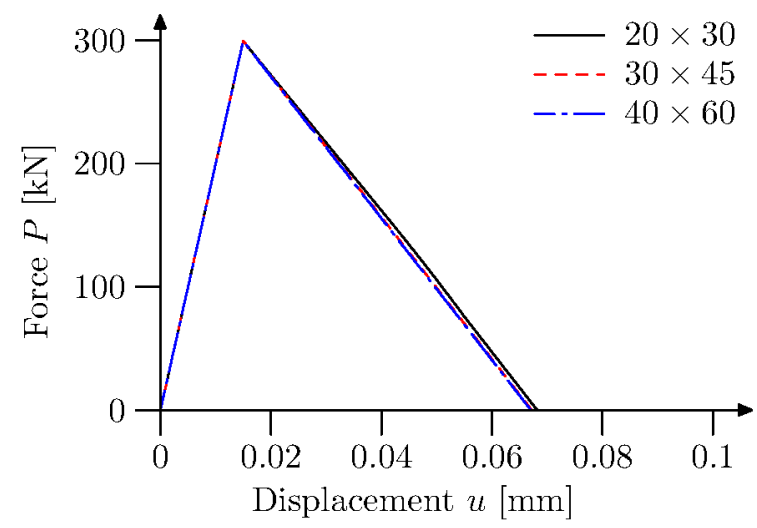

Figure 6: Uniaxial tension: force-displacement curves for different meshes.

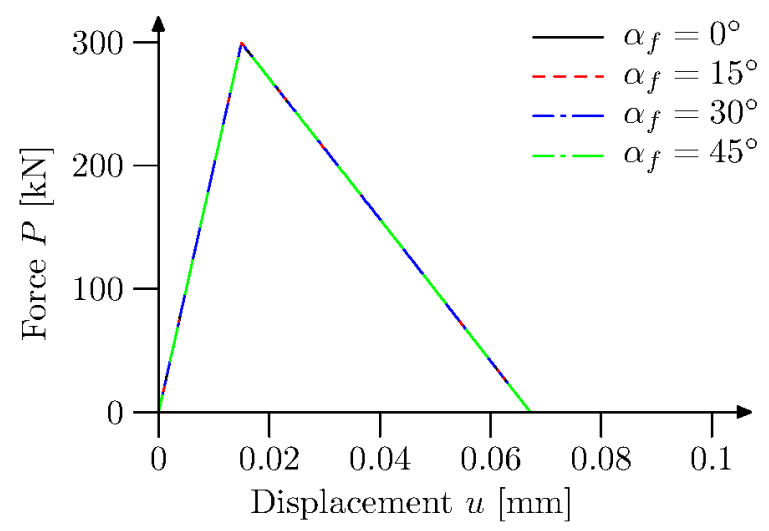

Figure 7: Uniaxial tension: force-displacement curves for different front angles $\alpha_{f}$.

\section{THREE-POINT BENDING}

The geometry of the concrete beam under plane stress conditions was given in Fig. 8. Due to a problem symmetry, a localized zone and discrete crack were formed along the vertical symmetry axis. The imposed final vertical displacement was $u_{f i n}=0.4 \mathrm{~mm}$. The Young modulus and Poisson ratio were assumed as $E=30 \mathrm{GPa}$ and $v=0.2$. The tensile strength was taken as $f_{t}=3.0 \mathrm{MPa}$. In plasticity, the exponential softening curve with the parameter $k_{u}=1.05 \cdot 10^{-3}$ was chosen. The characteristic length was equal to $l=10 \mathrm{~mm}$ and the non-locality parameter was $m=2$. In XFEM, the exponential softening scheme with the fracture energy $G_{f}=120 \mathrm{~N} / \mathrm{m}$ was assumed and the shear stiffness was $T_{s}=10^{12} \mathrm{~N} / \mathrm{m}^{3}$. The default transition point was $\kappa_{c d}=7.28 \cdot 10-3$ that corresponded to the $50 \%$ of the tensile strength. Three different finite element meshes were defined: coarse, medium and fine with 600, 1260 and 2280 4-node quad elements, respectively. In the mid-region, the refined meshes were assumed with 20, 40 and 80 elements along the vertical symmetry axis for a coarse, medium and fine mesh, respectively.

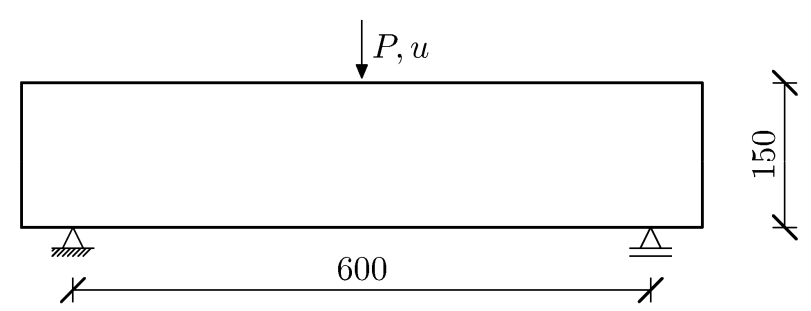

Figure 8: Three-point bending: geometry and boundary conditions.

Figure 9 presents the force-displacement curves for the different meshes and front angle $\alpha_{f}=15^{\circ}$. Only for the coarse mesh some force jumps were observed. For the finer meshes the curves were always smooth. The simulations with the angle $\alpha_{f}=0^{\circ}$ resulted in convergence problems with a fine mesh. It could be caused by the regular alignment of finite elements. The influence of the front angle $\alpha_{f}=15^{\circ}$ is depicted in Fig. 10. The simulations were performed with the medium mesh. For the front angle $\alpha_{f}=0^{\circ}$, the jumps were again observed (even larger than for the coarse mesh in Fig. 9). By taking non-zero values of the front angle $\alpha_{f}$, the bumps may be reduced/eliminated. The simulations were performed with parameters $\kappa_{0}$ and $\kappa_{1}$ chosen to define the transfer zone with the decrease of the tensile strength equal to $0.1 f_{t}$. Figure 11 shows the influence of the length of the transfer zone. When defining a longer transfer zone, a smoother force-displacement diagram was obtained, even when taking the front angle $\alpha_{f}=0^{\circ}$. 


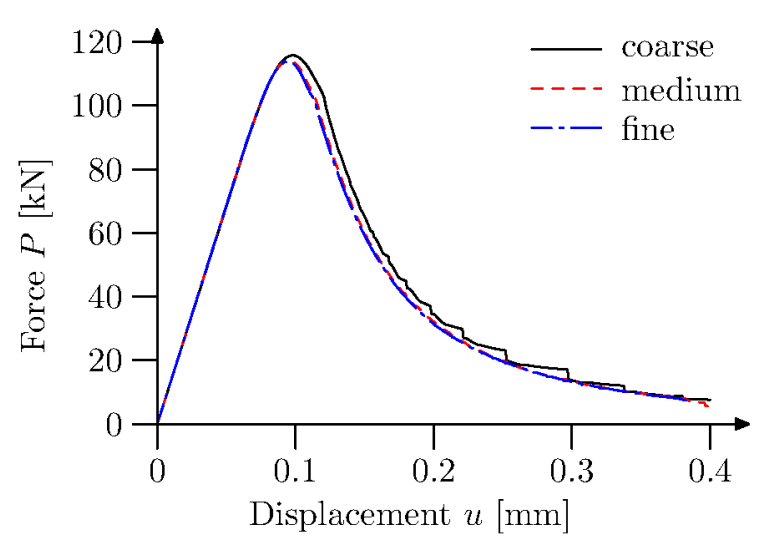

Figure 9: Three-point bending: force-displacement diagrams for different meshes.

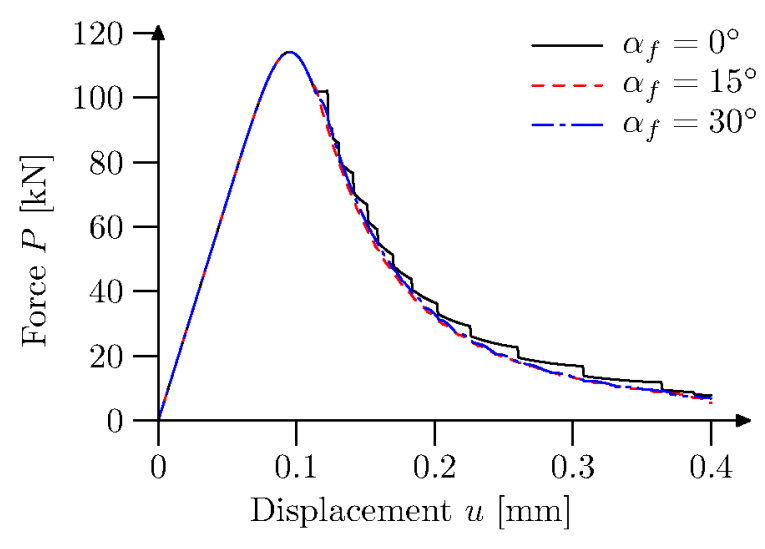

Figure 10: Three-point bending: force-displacement diagrams for different front angles.

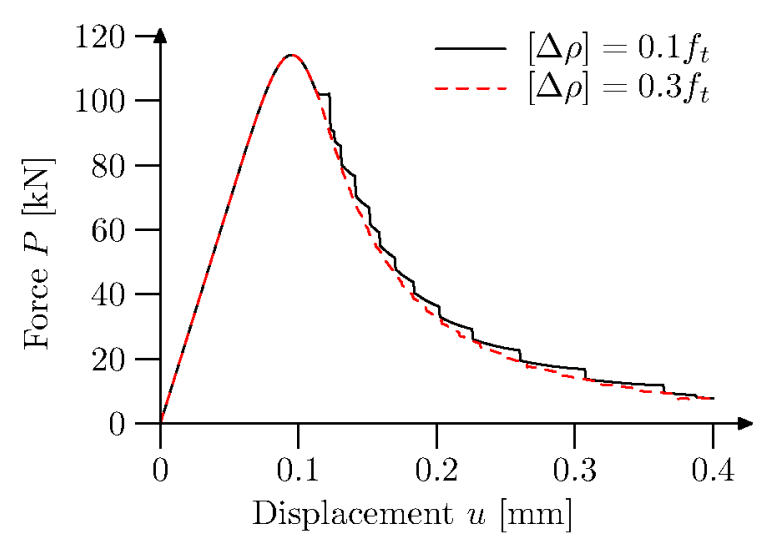

Figure 11: Three-point bending: force-displacement diagrams for different lengths of transition zone.

\section{NOORU-MOHAMED TEST}

Finally a double-edge notched (DEN) concrete specimen under combined shear and tension was analyzed (so-called the NooruMohamed test [13]). The length and height of the specimen were $200 \mathrm{~mm}$, and the thickness was $50 \mathrm{~mm}$ (Fig. 12). Two notches with the dimensions of $25 \times 5 \mathrm{~mm}^{2}$ were located in the mid-points of vertical edges. During the analyzed load scenario, the shear force $P_{s}$ was applied until it reached a specified value, while the horizontal edges were free. Then the shear force remained constant and the vertical tensile displacement was prescribed. In the experiment, two curved cracks with an inclination depending upon $P_{s}$ were obtained (for the small value of $P_{s}-$ the cracks were almost horizontal, for the large value of $P_{s}$ - the cracks were strongly curved), Fig. 13. In order to evaluate the quality of cracks, a measure called the crack height $h_{c}$ was introduced. It was defined as a vertical distance between a horizontal line connecting notches and the most far-distant point lying on crack lines. In the experiment, the height $h_{c}$, calculated as the average value for 4 cracks, was equal to $3.5 \mathrm{~cm}$ (in the range $2.7=4.3 \mathrm{~cm}$ ) for the horizontal shear force of $10 \mathrm{kN}$.

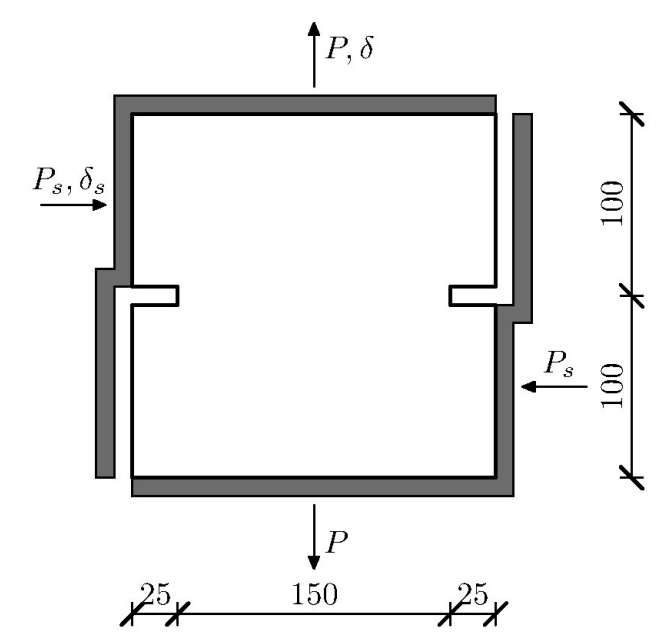

Figure 12: Nooru-Mohamed test: geometry and boundary conditions.

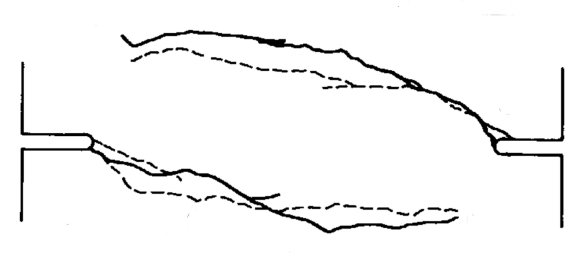

Figure 13: Nooru-Mohamed test: experimental crack pattern for $P_{s}=10 \mathrm{kN}$. 
The Young modulus and Poisson ratio were assumed as $E=32.8 \mathrm{GPa}$ and $v=0.2$. The tensile strength was taken as $f_{t}=2.3 \mathrm{MPa}$. In plasticity, the exponential softening curve with the parameter $k_{u}=4.20 \cdot 10^{-3}$ was chosen. The characteristic length was equal to $l=2 \mathrm{~mm}$ and the non-locality parameter was $m=2$. In XFEM, the exponential softening scheme with the fracture energy $G_{f}=75 \mathrm{~N} / \mathrm{m}$ was assumed and the shear stiffness was $T_{s}=10^{12} \mathrm{~N} / \mathrm{m}^{3}$. The default transition point was $\kappa_{c d}=2.91 \cdot 10-3$ (50\% of the tensile strength).

Figure 14 presents the computed forcedisplacement curves for 3 approaches. Each of them provided the results very similar to the

a)

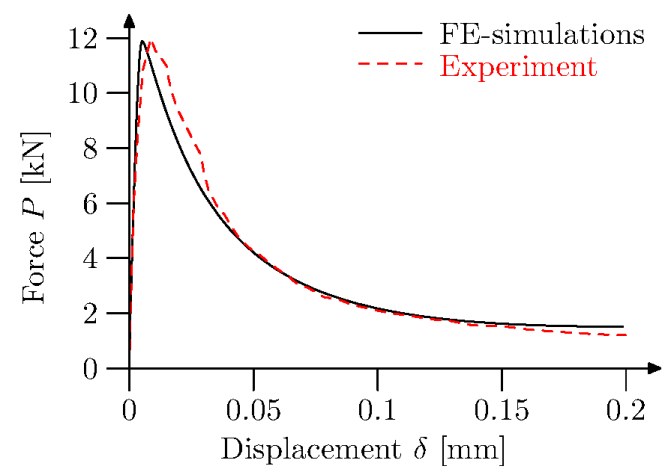

b)

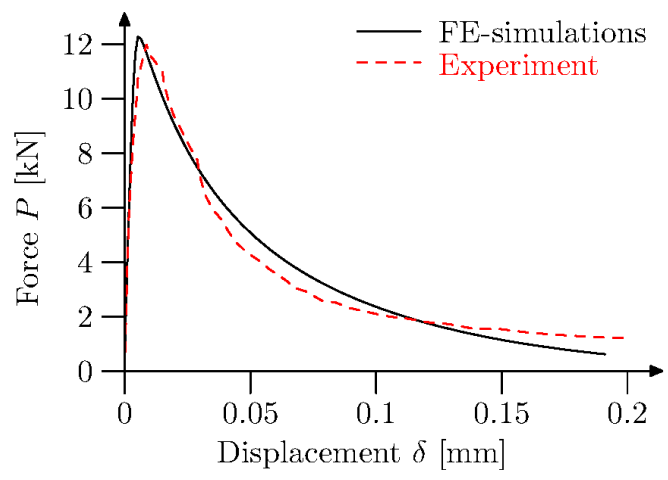

c)

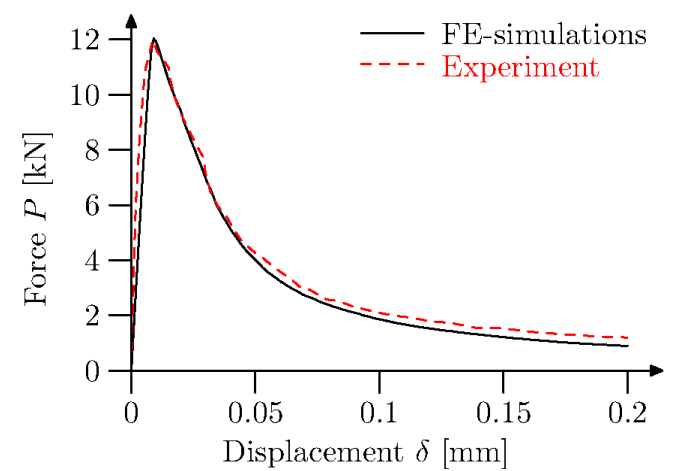

Figure 14: Nooru-Mohamed test: force-displacement curves for: a) pure elasto-plastic model, b) XFEM and c) coupled formulation. experiment. The calculated crack-patterns are depicted at Fig. 15. For the plasticity model the crack height $h_{c}$ was equal to $2.8 \mathrm{~cm}$ (slightly smaller than the experimental value of $3.5 \mathrm{~cm}$ ). In turn, the XFEM simulations

a)

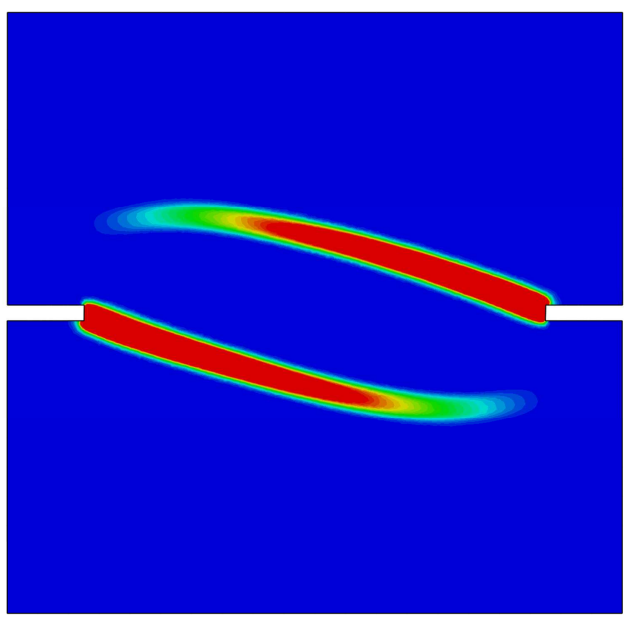

b)

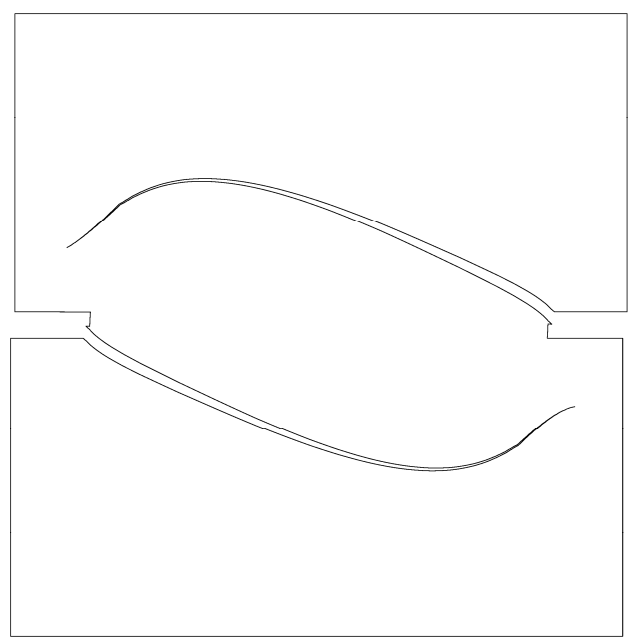

c)

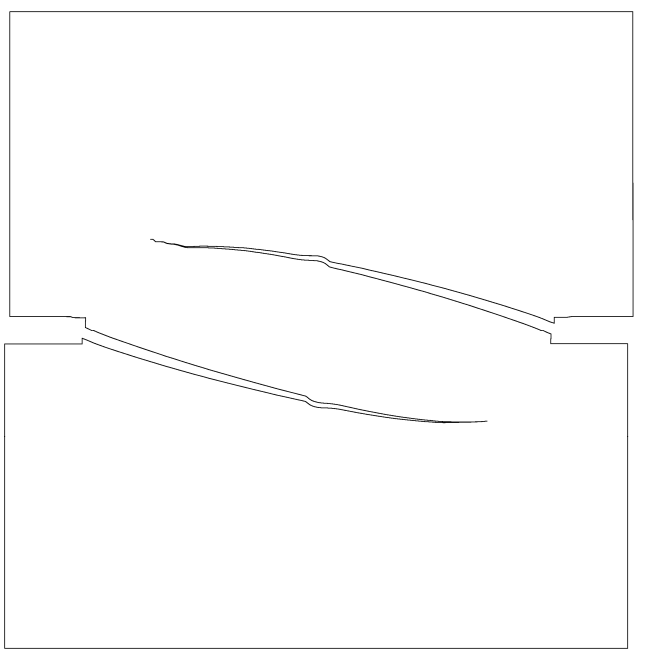

Figure 15: Nooru-Mohamed test: crack patterns for: a) pure elasto-plastic model, b) XFEM and c) coupled formulation. 
resulted in the too curved cracks (their height was $4.6 \mathrm{~cm})$. In the coupled approach the height $h_{c}$ was $2.8 \mathrm{~cm}$ (similar as in plasticity). Figure 16 shows the influence of the transition point. For the largest value of 5.82.10-3 (25\% of the tensile strength) some discrepancies with other curves were observed. In turn, the influence of the front angle was insignificant, (Fig. 17). The same conclusion was drawn for the influence of the length of the transition zone (Fig. 18).

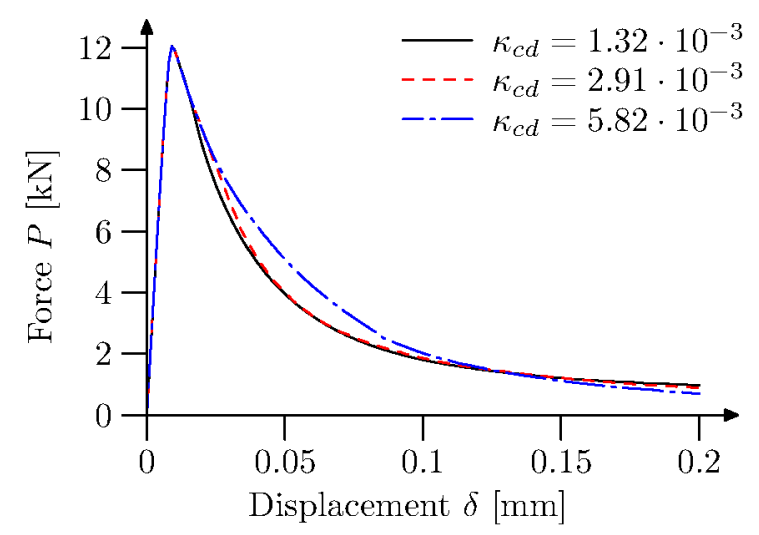

Figure 16: Nooru-Mohamed test: force-displacement curves for different transition points.

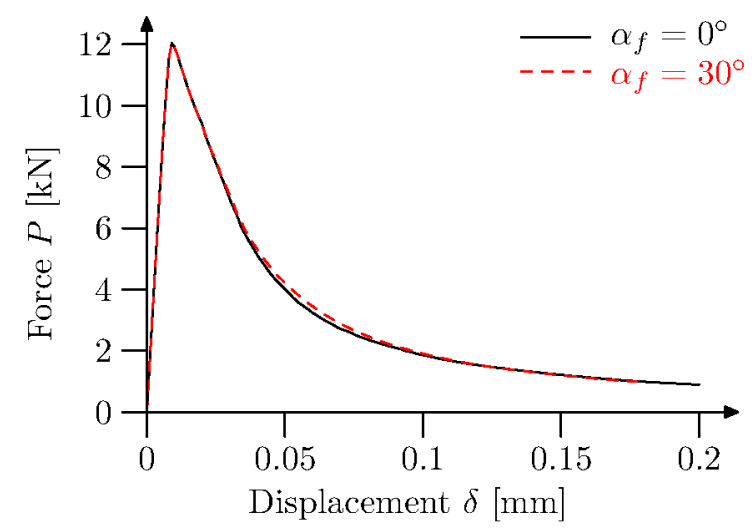

Figure 17: Nooru-Mohamed test: force-displacement curves for different front angles.

\section{CONCLUSIONS}

A coupled continuous-discontinuous constitutive model for concrete was presented. Its performance was tested under uniaxial and complex stress state conditions. The model was able to reflect basic properties of concrete specimens and to reproduce complex crack patterns during the Nooru-Mohamed test.

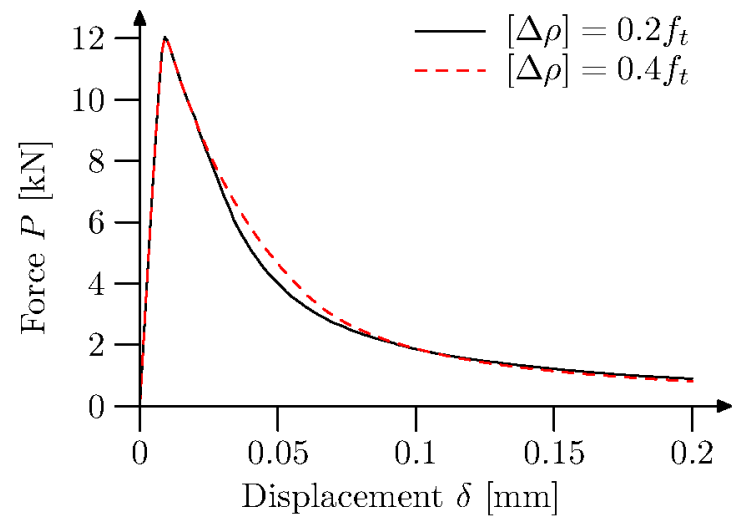

Figure 18: Nooru-Mohamed test: force-displacement diagrams for different lengths of transition zone.

The presented constitutive law enables a more realistic description of cracks in concrete elements. The formulation is general and it allows for using any constitutive laws in bulk continuum (e.g. isotropic version of a continuum damage mechanics) and any displacement jump-traction relationships along cracks. It may be also easily extended to take into account a decrease of a characteristic length upon loading and to obtain more realistic displacement profiles in localized zones.

Currently the comparison of results from 'pure' continuous and XFEM calculations and from simulations with the original coupled model is under way. In order to investigate the influence of the finite element size on the convergence and obtained results, the FEsimulations with other meshes will be also performed.

\section{ACKNOWLEDGMENTS}

Calculations were carried out at the Academic Computer Centre in Gdańsk.

\section{REFERENCES}

[1] Moës, N., Dolbow, J. and Belytschko, T. 1999. A finite element method for crack growth without remeshing. International Journal for Numerical Methods in Engineering 46:131-150.

[2] Simone, A., Wells, G.N. and Sluys, L.J. 2003. From continuous to discontinuous failure in a gradient-enhanced continuum damage model. Computer Methods in 
Applied Mechanics and Engineering 192:4581-4607.

[3] Comi, C., Mariani, S. and Perego, U. 2007. An extended FE strategy for transition from continuum damage to mode I cohesive crack propagation. International Journal for Numerical and Analytical Methods in Geomechanics 31:213-238.

[4] Wells, G.N., Sluys, L.J. and de Borst, R. 2002. Simulating the propagation of displacement discontinuities. International Journal for Numerical Methods in Engineering 53:1235-1256.

[5] Bobiński, J. and Tejchman, J. 2014. A constitutive model for concrete based on continuum theory with non-local softening coupled with eXtended Finite Element Method. In Bicanic, N., Mang, H., Meschke, G. and de Borst, R. (Eds) Computational Modelling of Concrete Structures, vol. I, pp. 117-126

[6] Bobiński, J. and Tejchman, J. 2016. A constitutive model for concrete based on continuum theory with non-local softening coupled with eXtended Finite Element Method. Finite Elements in Analysis and Design doi:10.1016/j.finel.2016.02.001.

[7] Hordijk, D.A. 1991. Local approach to fatigue of concrete. PhD Thesis, TU Delft.

[8] Brinkgreve R.B.J. 1994. Geomaterial models and numerical analysis of softening. PhD Thesis, TU Delft.

[9] Melenk, J.M. and Babuška, I. 1996. The partition of unity finite element method: basic theory and applications. Computer Methods in Applied Mechanics and Engineering 139:289-314.

[10] Wells, G.N. and Sluys, L.J. 2001. A new method for modelling cohesive cracks using finite elements, International Journal for Numerical Methods in Engineering 50: 2667-2682.

[11]Zi, G. and Belytschko, T. 2003. New crack-tip elements for XFEM and applications to cohesive cracks. International Journal for Numerical Methods in Engineering 57:2221-2240.

[12] Cox, J.V. 2009. An extended finite element method with analytical enrichment for cohesive crack modelling.
International Journal for Numerical Methods in Engineering 78:48-83.

[13] Nooru-Mohamed M.B., 1992. Mixed mode fracture of concrete: an experimental research. $\mathrm{PhD}$ Thesis, TU Delft. 